\title{
An Accurate Optic Disc Segmentation from Peripapillary Atrophy Incident Retinal Images
}

\author{
M. R. N. Tagore \\ Department of Electronics \& \\ Communication Engineering \\ Vasireddy Venkatadri Institute \\ of Technology \\ Nambur, Guntur, A.P, India
}

\author{
E. V. Krishna Rao, PhD \\ Department of Electronics \& \\ Communication Engineering \\ Lakireddy Bali Reddy College \\ of Engineering, \\ Mylavaram, A.P, India
}

\author{
B. Prabhakar Rao, PhD \\ Department of Electronics \& \\ Communication Engineering \\ JNTU Kakinada, \\ Kakinada, A.P, India
}

\begin{abstract}
Glaucoma detection system analyses the retinal images. This system gives vital information about presence of Glaucoma, the second largest cause of blindness. Usually the Glaucoma patients have large Cup-to-Disc ratio (CDR), inspection of CDR of Optic Disc is crucial of any Glaucoma detection system. Therefore accurate segmentation Optic Disc (OD) and Cup are critical in the formulation of CDR. Current work focuses on OD segmentation to develop an efficient Glaucoma detection system. A serious difficulty arises in OD segmentation in retinal images having inhomogenities due to Peripapillary Atrophy (PPA) and fuzzy boundaries. So this paper proposes a OD Segmentation method that takes care of fuzzy boundaries and inhomogenity presenting in the retinal images. At First, The matched filter with Cauchy kernel is applied to extract blood vessels. Then Vessel Directional Matched filter(VDM) is used to locate the centre of the optic disc approximately followed by Vessel Inpainting to erase the vasculature in the OD region. Finally a LBF energy based active contour model is formulated that embeds edge and region based information in the newly formulated locally computed signed pressure force(SPF) function, to segment OD. The obtained results indicate that the proposed OD segmentation outperforms many existing methods.
\end{abstract}

\section{Keywords}

Retinal Image, Glaucoma, Level Sets, Optic Disc Segmentation

\section{INTRODUCTION}

The algorithm proposed in this paper is the prime component of the system that detects and grades diabetic retinopathy (DR) and more importantly it is critical sub-system in glaucoma assessment. The DR is a familiar complication resulting of diabetes and is the main cause of vision loss in many developing nations [1]. The loss of vision can be prevented in at least $60 \%$ cases if an appropriate treatment can be given at right time. So the screening plays vital role in detecting retinopathy. As per the studies available over 80 million persons across the globe are susceptible to glaucoma by $2020[2-3]$. This is a chronical, irreversible and asymptomatic disorder which will damage Optic Nerve Head progressively which leads to deteriorating visual perception and finally to loss of vision. During initial stages the patients are unaware of its presence until a perceptible degradation in vision has developed at a later stage; hence it acquires the tag of 'silent thief of sight'. It is a widespread belief that the Cupto-Disc ratio which is used as measure to detect Glaucoma is strongly related to Peripapillary atrophy (PPA) [4]. Usually huge number of retinal images has to be screened by
Ophthalmologists. These screening procedures require lot of skilled expertise to grade retinal images which is time consuming and often error prone. Reliable automated systems that can accurately segment the optic disc in the presence of PPA have tremendous significance in the design of a computer aided diagnosis for glaucoma.

\subsection{State of the Art}

Though the features of OD are well-defined, the OD localization $\mathrm{n}$ in a robust manner is not that simple. The prime reason for this is appearance of pathologies. As a consequence many methods have been proposed for OD detection by considering differences in brightness, size, location and appearance among various images.

Usual OD region has yellowish appearance due to emergence of dark (red-filled) blood vessels along with whitish nerve fibers. This feature is well exploited by Sinthanayothin et. al. [5] to detect OD successfully. The intensity component is obtained first which then the intensity component is processed using adaptive local contrast enhancement prior to OD segmentation. The OD detection is performed using a window of size equal to OD with in which a region of highest intensity variation among adjacent pixels is detected.

The same brightness variance feature is effectively utilized by Li and Chutatape et. al [6] and developed a model based approach to extract all OD candidate regions. Here they have rejected the $99 \%$ pixels which represent lowest intensities from the intensity image of the given fundus image. The remaining $1 \%$ pixels are definitely belongs to either OD or bright lesions. These pixels are clustered then and simple clusters are removed. Principal Component Analysis is used on 10 training images manually cropped square OD regions from intensity images to develop OD model. A PCA transform at various scales is applied through a window on each pixel of the candidate regions and the OD is located from the region which has smallest Euclidean distance from its projection to disc space. The general notion regarding DR is the pathologies like exudates are small in size when compared to OD though similar in brightness. Hence, instead of using variation of intensity as a criterion to localize OD, Walter and Klein [7] identified the OD center by detecting the center portion of the largest yellowish portion of the given image. A binary image is obtained by them which include all bright regions. Many approaches that are found in literature will mostly comprise three different models for optic disc extraction: level set based contour detection, pixel classification and graph search [8-11]. Morales et al [12] have used mathematical morphology and principal component analysis (PCA) based method together inorder to extract OD 
contour. They have employed different procedures such as geodesic distance transformation, generalized distance function, and stochastic watershed for segmenting OD. A novel multiresolution sliding band filter is proposed by Dashtbozorg et al. [13] to segment OD. The authors have chosen high and low resolution sliding band filters out of which a set of maximum response pixels are obtained. These pixels will give the near estimate of the OD contour. The accuracy of OD segmentation is severely affected by inhomogenity due to PPA.

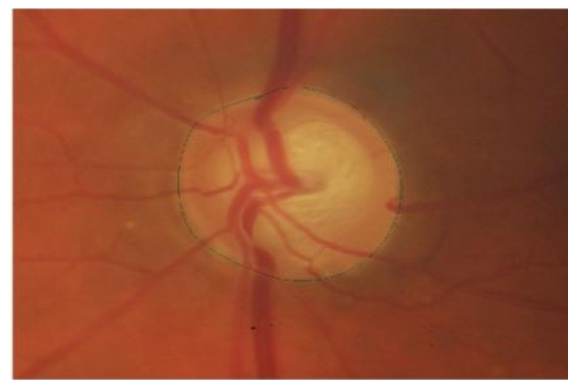

Fig 1: A color retinal image overlaid with green colored manually segmented OD boundary. This is a OD centric image.

The prime objective of this work to provide rich descriptions of Optic Disc segmentation which can be used for different retinal disease assessment techniques. The main components and contributions of the present work are: a) Vasculature extraction is done using non-gaussian kernel based matched filter b) Residual vessels in OD are erased using Vessel Inpainting. c) Finally an implicit local region and edge information based active contour model .Our work is organized as follows: We describe the method of OD segmentation system in Section 2 Section 3 describes the detailed evaluation of this work on a set of 59 retinal images and conclusions are presented in Section 3.

\section{METHODS}

The precise segmentation of OD is helped by elimination of vasculature in OD region as their presence within the OD affect the boundary detection. Hence, if vasculature are extracted first and erased prior to the OD detection accuracy of segmentation can be improved. In this section the

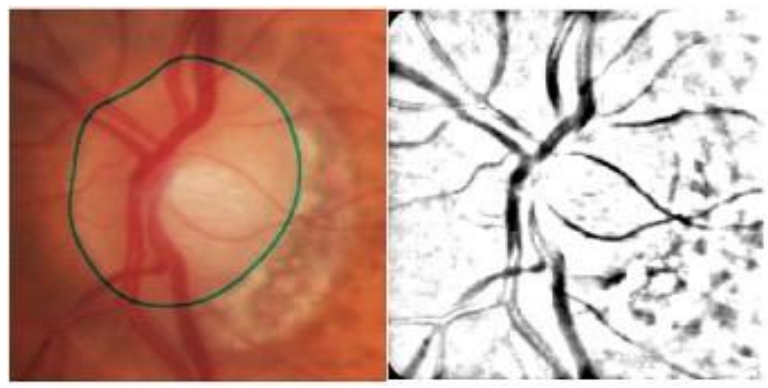

segmentation of retinal vasculature and OD and vessel erasing through inpainting are presented.

\subsection{OD Segmentation}

\subsubsection{Vasculature Segmentation}

There would be many instances where the retinal images suffer with poor contrast due to non-uniform illumination. A shade correction technique is used as given in [14] to perform illumination correction before vessel detection. Generally, most of the vessel detection algorithms consider gaussian function as the kernel for matched filter to estimate the vessel profile. But, they have conveniently ignored the fact that not all vessel profiles are truly gaussian. Some wide and large blood vessel segments often have non-gaussain intensity profiles. Hence, in this work we have used Cauchy kernel to enhance blood vessel segment structures which will fit better than its gaussian counterpart. The second order Cauchy kernel [15] in one dimension is defined as

$$
f(x)=\frac{1}{\pi \gamma\left[1+\left(\frac{\left(x-x_{o}\right)}{\gamma}\right)^{2}\right]}
$$

where ' $x_{o}$, is a scaling parameter and $\gamma_{\text {is scaling }}$ parameter. The $\gamma_{\text {in Cauchy is similar to }} \sigma_{\text {in gaussian }}$ kernel. A small value of $\gamma_{\text {will lead to narrow curve and vice- }}$ versa. By choosing a non-zero value for ${ }^{x_{o}}$ the pdf curve can be shifted along horizontal axis. In the present work $x_{o}=0$ selected. We have used a set of rotated Cauchy kernels as the blood vessels are oriented in many directions in a given retinal image.

This implementation uses 12 matched filter orientations an increment of 15-degree. After the main vascular structures are detected, histogram clustering based on Hierarchical thresholding [16] is applied to distinguish enhanced vessels against the image background. All the phases and results of blood vessel segmentation are shown in Fig. 2.

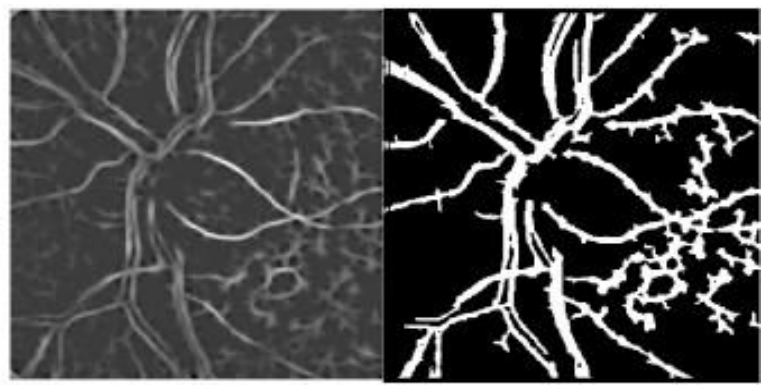

Fig 2: Phases of vasculature extraction (a) Original fundus image with groundtruth (b) Result of Shade correction to remove non-uniform illumination(c) Cauchy kernel based Matched filtering is applied to detect blood vessel structure (d) Final vessel structure after applying histogram clustering based on Hierarchical thresholding

\subsubsection{OD Localization}

For OD segmentation location of OD is to be defined first. The process of localizing OD region uses convergence feature of blood vessels into OD region used. After locating OD we can extract the region of interest. We have used vessel direction matched filter which is a powerful technique to locate OD [17]. The vessel direction matched filter is modified as shown in Fig.3 to fit to present database.

The vessel direction matched filter of size $11 \mathrm{X} 11$ is resampled to sizes $295 \times$ 99, 441 X 148, 587X197 and 735X 245 using bilinear interpolation. These four filters are applied simultaneously, then difference between the four filter responses is computed and the difference between the four 
responses is determined. The pixel with smallest difference should be center of optic disk. The OD extraction is facilitated by localization of OD. A vessel erasing step[23] is performed in order to remove vessels at OD boundary, results are given in Fig.4.

\subsubsection{Contour Detection of $O D$}

OD segmentation is a demanding task as the shape of the OD varies from image to image, depth or colour variation of the OD which is a sign of presence of retinal pathologies, like glaucoma, instantiation of smooth transition of OD at boundary regions and formation of Peripapillary Atrophy .The accurate segmentation OD will be very much useful to study the state and progression of various eye diseases like glaucoma. It also useful in analyzing the end treatment results of a unhealthy person. As already discussed the OD

$\begin{array}{lcccccccccc}135 & 120 & 120 & 105 & 105 & 90 & 75 & 75 & 60 & 60 & 45 \\ 150 & 135 & 120 & 120 & 105 & 90 & 75 & 60 & 60 & 45 & 30 \\ 150 & 135 & 120 & 105 & 105 & 90 & 75 & 60 & 45 & 45 & 30 \\ 165 & 150 & 135 & 120 & 120 & 90 & 60 & 60 & 45 & 30 & 15 \\ 165 & 165 & 150 & 135 & 135 & 90 & 45 & 45 & 30 & 15 & 15 \\ 0 & 0 & 0 & 0 & 0 & 90 & 0 & 0 & 0 & 0 & 0 \\ 15 & 15 & 30 & 30 & 45 & 90 & 135 & 150 & 150 & 165 & 165 \\ 15 & 30 & 45 & 60 & 60 & 90 & 120 & 120 & 135 & 150 & 165 \\ 30 & 45 & 60 & 60 & 75 & 90 & 105 & 120 & 120 & 135 & 150 \\ 30 & 45 & 60 & 75 & 75 & 90 & 105 & 120 & 135 & 135 & 150 \\ 45 & 60 & 60 & 75 & 75 & 90 & 105 & 105 & 120 & 120 & 135\end{array}$

Fig 3: Vessel Directional Matched Filter's base kernel of size 11X11.

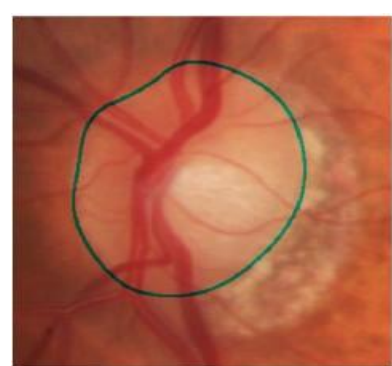

(a)

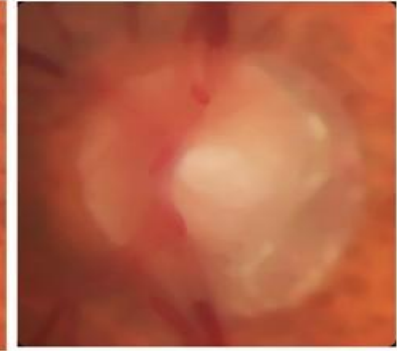

(b)
Fig 4: (a) OD centric original retinal image. (b) Vessel inpainting result. After performing vessel directional matched filtering on the original image vessel inpainting is performed to erase blood vessels.

The edge indicator function of LFI energy is replaced with region based information which is extracted by LBF model using image information to enable segmentation of OD boundary in retinal images having inhomogeneity and fuzzy boundaries. The area regularization term uses this region into LBF energy model. segmentation is really challenging for the instances like smooth boundary transitions and formation of intensity inhomogenity due to PPA incidence.

A novel implicit region based OD detection active contour model that uses edge information is proposed to improve OD segmentation over a wide variety of OD instances .Local Image fitting(LIF) and Local Binary fitting(LBF) energy models $[18,19]$ is instrumental tools behind the proposed active contour model. This integrates both LIF and LBF energy models by formulating a locally computed mean value based signed pressure force function (SPF). Essential strength of this method is that it is not imposed any shape based constraints. A method is formulated by embedding LFI model

based information, thus the contour will be forced to stop even at weak and blurry boundary whereas the length regularization term uses edge information that accelerates the contour towards boundary.

A novel implicit region based OD detection active contour model that uses edge information is proposed to improve OD segmentation over a wide variety of OD instances .Local Image fitting(LIF) and Local Binary fitting(LBF) energy models $[18,19]$ is instrumental tools behind the proposed active contour model. This integrates both LIF and LBF energy models by formulating a locally computed mean value based signed pressure force function (SPF). Essential strength of this method is that it is not imposed any shape based constraints. A method is formulated by embedding LFI model into LBF energy model. The edge indicator function of LFI energy is replaced with region based information which is extracted by LBF model using image information to enable segmentation of OD boundary in retinal images having inhomogeneity and fuzzy boundaries. The area regularization term uses this region based information, thus the contour will be forced to stop even at weak and blurry boundary whereas 
the length regularization term uses edge information that accelerates the contour towards boundary.

The energy functional is contribution of three terms as given below.

$$
E=E_{d t}+v E_{\mathrm{lg}}+u E_{a r}
$$

Where the data term $E_{\mathrm{dt}}$ carries image information, $\lambda$ and $\mathrm{u}$ are two scalar constants while $\lambda$ is always positive and $u$ can be either positive or negative depends on whether the initial contour outside the object or inside the object. The $\mathrm{E}_{\mathrm{d}} \mathrm{t}$ is similar to data fitting term of $\mathrm{C}-\mathrm{V}$ model, while Ear and $\mathrm{E}_{\mathrm{lg}}$ are energy terms penalizing the length and area regularizations. Many implementations have richly dealt with data fitting terms while the regularization terms $E_{a r}$ and $E_{l g}$ have given less attention. In equation (2) $E_{l g}$ is edge-based length term and Ear is region-base area term. The $\mathrm{E}_{\mathrm{lg}}$ and Ear are computed as follows.

$$
E_{\mathrm{lg}}(\phi)=\int_{\Omega} g(I) \delta(\phi)|\nabla \phi| d x
$$

Here $g(I)$ is the edge indicator function and $\delta(\phi)$ is the first derivative of Heaviside $H(\phi)$ which are given below.

$$
\left\{\begin{array}{l}
\delta(l)=\frac{\varepsilon}{\pi\left(l^{2}+\varepsilon^{2}\right)} \\
H(\phi)=\frac{1}{2}\left(1+\frac{2}{\pi} \arctan \left(\frac{l}{\pi}\right)\right)
\end{array}\right.
$$

The edge indicator function $g(I)$ is strictly monotonically decreasing function which is allowed to take positive values as indicated in the subsequent equation.

$$
g(I)=\frac{1}{1+\left|\nabla g_{\sigma} * I\right|^{2}}
$$

The present implementation does not require any reinitialization; the initial level set function $\phi_{o}$ is taken as

$$
\phi_{o}=\phi(x, t=0)=\left\{\begin{array}{lr}
-1 & \mathrm{x} \in \Omega_{o}-d \Omega_{o} \\
0 & d \Omega_{o} \\
+1 & \mathrm{x} \in \Omega_{o}-d \Omega_{o}
\end{array}\right.
$$

The region based area term function is given by

$$
E_{a r}(\phi)=\int_{\Omega} S(I) H(-\phi) d x
$$

Where the $\mathrm{S}(\mathrm{I})$ is locally generated image through signed pressure force function(SPF) which is obtained as follows

$$
S P F(I)=S(I)= \begin{cases}\frac{I(x)-I_{L F I}}{\max \left(\left|I(x)-I_{L F I}\right|\right)}, & \mathrm{I}(\mathrm{x}) \neq 0 \\ 0 \quad, & \mathrm{I}(\mathrm{x})=0\end{cases}
$$

Obviously this SPF is formulated based on locally computed mean value. This energy functional is acquiring a minimum value at the OD boundary.

\section{EXPERIMENTAL EVALUATION}

Fifty nine images are acquired from databases DIARETDB0 [20] and RIMONE [21]. The database of fifty nine colour retinal images is used to test the competency of the proposed OD segmentation system. Among these 59 images 17 are normal and 42 are glaucomatous images with different grades of PPA. These images are resized to squares of $1024 \times 1024$ pixels as these testing images are acquired from multiple sources. There are no ground truths of OD available for retinal images of DIARETDB0 database. All the fundus images which have no ground truths have been annotated by an expert ophthalmologist. The other models implemented for OD segmentation are LIF energy model[19], Chan - Vese (CV) energy model as implemented in[18] , and modified LBF[23] which uses multiple image plane information energy model as implemented in [8] active contour models. The parameter selection for all implementations is illustrated in Table 1.

\subsection{Distance Measures}

\subsubsection{Hausdorff Distance}

Hausdorff distance $\mathrm{H}$ is the maximum value of the set $\{\mathrm{Hsg}$, $\mathrm{Hgs}\}$.The Hsg is the directed distance between $\mathrm{S}$ and $\mathrm{G}$ and oppositely directed distance is Hgs . For every pixel 'p' of $S$ we compute set of minimum distances between $\mathrm{p}$ and each pixel of $\mathrm{G}$. The maximum distance of this minimum distance set is Hsg . The reversal distance is Hgs .

\subsubsection{Dice Coefficient $\left(C_{D}\right)$}

The Dice coefficient tries to extract spatial overlap and spatial similarity between two binary images. It is a more general metric with a ' 0 ' stand for absolutely zero overlap and ' 1 ' indicates perfect overlap between the two images. In the present work the $\mathrm{C}$ will be expressed in percentage as indicated below.

$$
C_{D}=\frac{2|S \cap G|}{|S|+|G|} X 100
$$

\subsubsection{False Positive and False Negative Dice}

To make accurate analysis and to perform robust evaluation we will obtain false positive dice(FPD) and false negative dice(FND) as given below which are the measures of over and under segmentations respectively.

$$
\begin{aligned}
& F P D=\frac{2|S \cap \bar{G}|}{|S|+|G|} X 100 \\
& F N D=\frac{2|\bar{S} \cap G|}{|S|+|G|} X 100
\end{aligned}
$$

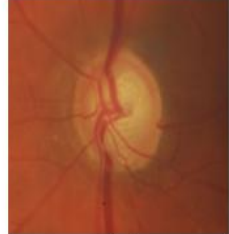

(a)

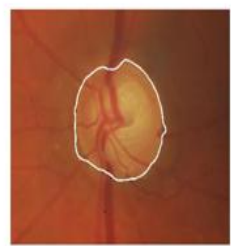

(d)

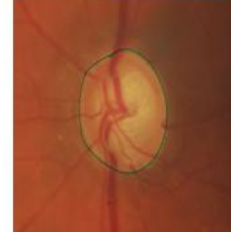

(b)

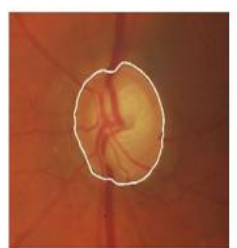

(e)

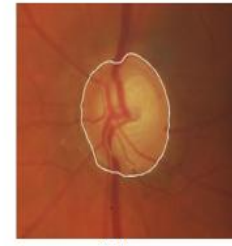

(c)

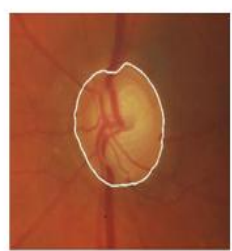

(f)
Fig 5: Segmentation results of Normal retinal image: (a) Original image; (b) Ground truth impinged on original image; (c),(d),(e) segmentation results of LIF,C-V and modified LBF model ;(f) is the detected OD boundary of the proposed method. The segmentation results of all models appear to be visually similar. 
Table 1: Parameter selection for various active contour models: Here $\sigma 1$ and $\sigma 2$ are Standard deviations of Gaussian kernels. $\lambda 1$ and $\lambda 2$ are the two positive constants defined in $C-V$ model. The $\mu$ is another constant selected exclusively for CV and LBF models which can be positive or negative depending on initializing the initial contour inside or outside the object boundary to

be segmented for $\mathrm{C}-\mathrm{V}$. In the proposed model uses the parameter $\mathrm{u}$ to emulate the parameter $\mu$.The $v$ is the force term parameter and $\epsilon$ is a positive constant used in Dirac and Heaviside function approximations, as defined in proposed method.

\begin{tabular}{ccccc} 
Parameter & LIF & C-V & LBF & Proposed Method \\
$\lambda_{1}$ & - & 1 & 1 & 1 \\
$\lambda_{2}$ & - & 1 & 1 & - \\
$\mu$ & - & 0.2 & 1 & 3 \\
$\sigma_{1}$ & 5 & - & 4 & 22 \\
$\mathrm{v}$ & - & 1 & $0.001 X 255^{2}$ & 1.5 \\
$\epsilon$ & 1.5 & 1.5 & 1.5 & 1 \\
$\sigma_{2}$ & 1 & - & - & 0.8 \\
$\mathrm{u}$ & - & - & - & \\
\hline
\end{tabular}

\subsubsection{Correlation Coefficient}

The fourth is the correlation coefficient and it is defined as

$$
\rho(x, y)=\frac{\operatorname{cov}(X, Y)}{\sigma_{x} \sigma_{y}}=\frac{E\left[\left(X-\mu_{x}\right)\left(Y-\mu_{y}\right)\right]}{\sigma_{x} \sigma_{y}}
$$

A value for $\rho$ equal to ' 1 ' indicates variables $\mathrm{X}$ and $\mathrm{Y}$ are perfectly positively correlated; value of ' -1 ' means that there exists a perfect opposite correlation between the variables, and a value ' 0 ' indicates that the variables are not at all correlated.

The image of the Fig.5.a is an example normal retinal image which has well defined OD shape compared to others. The segmentation results of all the four different contour models are nearly similar. The first image in Fig.8.a is having little illshaped OD with persisting gradient variations. As LBF and LIF models are less subtle to these local gradient variations, they detect most of the boundary region except certain portions where gradient variations are predominant. On the other hand it is evident that the CV model will handle these variations very well compared to LBF and LIF models but the low intensity levels in the vicinity of the bright regions are not keenly picked up by this model. Our proposed OD boundary segmentation method is exhibiting high accuracy in successfully segmenting the OD even in regions intensity flatness. The second row Fig .6 has a healthy retinal image with fuzzy boundary. In this scenario the results of segmentation clearly indicates that the proposed OD segmentation method better captures the contour of the OD. The segmentation results of images that are affected by Peripapillary atrophy are presented in the last two rows. The third row of Fig.6 is an example of an image, severely affected by PPA whereas the retinal image in last row indicates a moderate PPA instance.

Table 2: Performance indicators of Optic disc Segmentation

\begin{tabular}{lllllll}
\hline Model & $\begin{array}{l}\text { Dice } \\
\text { Coefficient } \\
\text { (CD) }\end{array}$ & $\begin{array}{l}\text { Hausdorff's } \\
\text { Distance(H) }\end{array}$ & $\begin{array}{l}\text { False Positive } \\
\text { Dice(FPD) }\end{array}$ & $\begin{array}{l}\text { False Negative } \\
\text { Dice(FND) }\end{array}$ & $\begin{array}{l}\text { Correlation } \\
\text { coefficient }\end{array}$ & $\begin{array}{l}\text { Average } \\
\text { time } \\
\text { image(s) }\end{array}$ \\
\hline LIF[28] & 86.5 & 24 & 36 & 34 & 0.672 & 33 \\
CV[31] & 78 & 12 & 38 & 32 & 0.562 & 41.5 \\
LBF[32] & 91 & 23 & 24 & 31 & 0.785 & 46 \\
$\begin{array}{l}\text { Proposed } \\
\text { Method }\end{array}$ & 93 & 9 & 18 & 21 & 0.93 & 44 \\
\hline
\end{tabular}




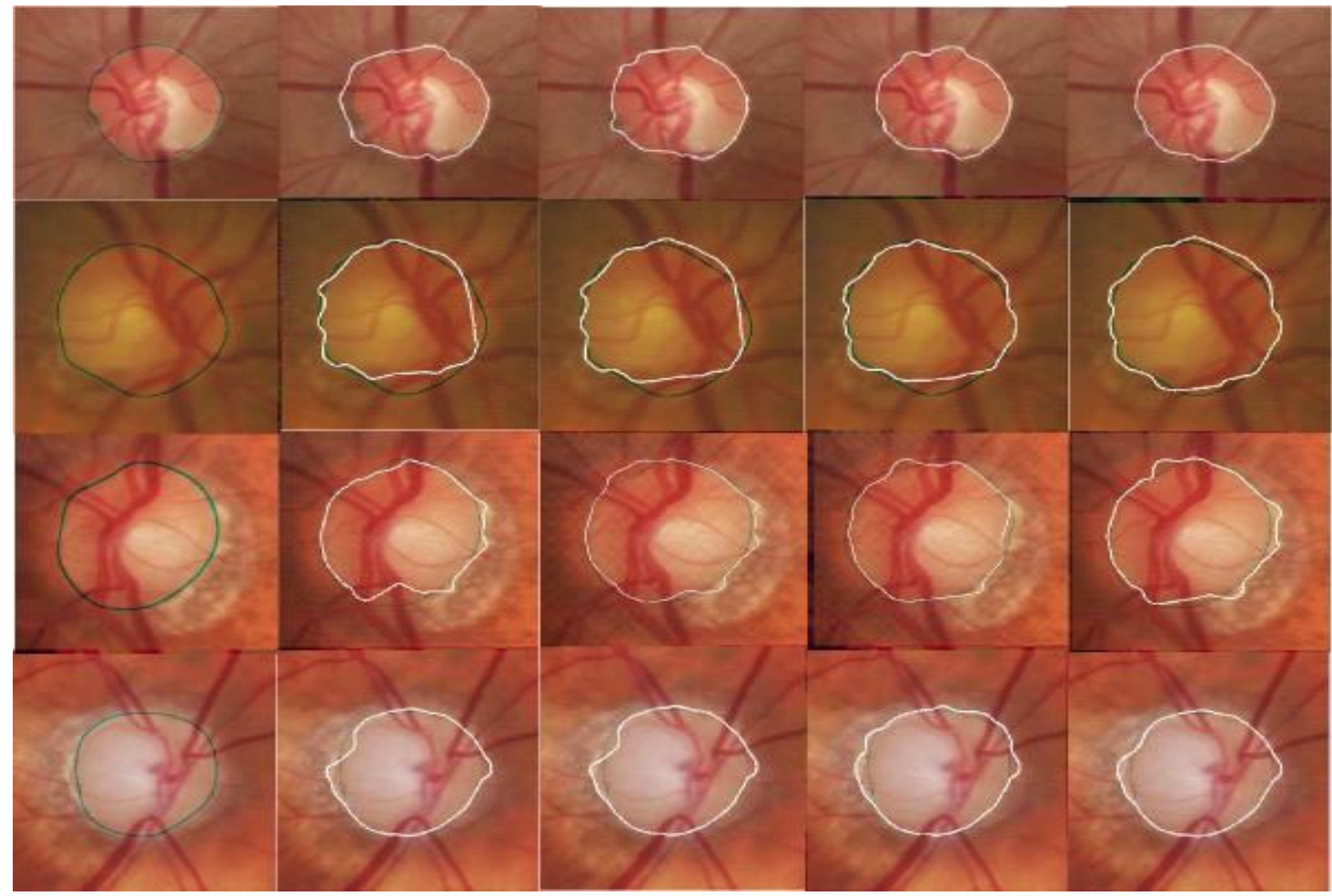

Fig 6: Results of OD segmentation results for 4 images are given here. The First column is having original colour fundus images overlaid with ground truths; Second column: OD segmentation results of LFI active contour model [28]; Third column: OD segmentation results of CV energy model as implemented by Joshi et al[31]; Fourth column: modified LBF energy model results as implemented by Kande et al.2016 [32] ; Fifth column: proposed method results. Here each image is having OD boundary encircled with Green colour and white coloured contour. The green coloured contour in each image indicates the OD boundary as annotated by the expert and white colour indicates obtained contour. The last two rows show the retinal images and segmentation results that are examples of severe atrophy conditions.

As the PPA introduces intensity inhomogenity regions surrounding optic disc, the $\mathrm{CV}$ model which assumes homogeneous conditions throughout the given image and utilizes gradient intensity information of image only, it may not segment OD accurately under PPA instances. At the same time the LIF model uses global region information for the level set evolution, it cannot detect all object boundaries accurately under these conditions of PPA as indicated in 2nd and 3rd columns of last two in Fig. 6. Obviously the misdetection may occur at the stronger edges of atrophy which can be detected as the boundary of OD. The fourth column of Fig. 6 are the results of modified LBF model as implemented in [23] indicates that their method is more accurate than LIF and CV based models under intensity inhomogeinity conditions though it is computationally complex. However the segmentation results this model can be more vulnerable to image contrast. This is clearly visible in fourth column of third row where left half of the image is suffered with contrast variations. By contrast, our method uses newly formulated locally computed mean based SPF, can successfully segment inhomogeneous boundary regions accurately which is independent of contrast variations across the object boundary. It can be seen from the segmented images of last two rows of Fig.6 apart from proposed method all other methods failed to detect boundary accurately because of fuzziness in the edge of normal OD or fuzzy OD with PPA. Comparison of proposed boundary segmentation method of OD with three other prominent OD segmentation methods is presented in Table 2.The comparison is performed in terms of average Dice Coefficient, average Hausdorff's Distance, average false positive and false negative dices and average correlation factor. The present OD segmentation method achieved best $C D$ score indicating the robustness over other methods. The computed dice coefficient of LBF based method is similar to proposed method but it gives large false negative dice. The proposed method achieved highest correlation coefficient making it superior over the other methods. This improved performance can be attributed to reduction in FPs as it is using both local region and edge based information. The Hausdorff's Distance achieved by the proposed method is lowest among all methods. There exists high proximity between segmented OD using proposed method and manually annotated ground truth as indicated by the average correlation of 0.93 .

After thorough experimentation, the results of the proposed method in comparison with those of the LBF model base method, CV Model based method and LIF based methods, have exhibited high accuracy consistently in OD segmentation. Though our method is highly responsive when OD boundary region is surrounded by inhomogeneous intensity regions, it needs more processing time compared with other active contour models as local mean values have to be computed.

\section{CONCLUSION}

The focus of the present work is to device an effective methodology to segment the OD from 2D retinal images. Vessel directional matched filter is applied to localize OD. Our approach of OD segmentation is edge and region information based active contour model that uses locally computed SPF function based LBF energy model. Thus it can 
avoid the inhomogenity like PPA regions formed outside the OD. At the same time our method is insensitive to initial contour as well. A minor deficiency of this method is at times cup boundary may be detected as OD contour because of noise introduced due to fake edges and it requires more computational time as well. We computed four distance measures and three Figures of merit to validate our method. The results of proposed SPF based OD segmentation method are superior to some of the state-of-the-art methods. To sum up, our method is advantageous over gradient based active contour models, LIF models while segmenting normal, fuzzy, irregular Optic disc shapes.

\section{REFERENCES}

[1] D. E. Singer, D.M. Nathan, H. A. Fogel, and A. P. Schachat, "Screening for diabetic retinopathy," Ann. Int. Med., vol. 116, pp. 660-71, 1992.

[2] Quigley, H.A., Broman, A.T., 2006. The number of people with glaucoma worldwide in 2010 and 2020. Br. J. Ophthalmol. 90, 262-7

[3] Kass, M.A., Heuer, D.K., Higginbotham, E.J., et al., 2002.The Ocular Hypertension Treatment Study: a randomized trial determines that topical ocular hypotensive medication delays or prevents the onset of primary open-angle glaucoma. Arch. Ophthalmol. 120,701-13, discussion 829-30.

[4] E. M. Kohner, Diabetes and the Eye, Oxford Textbook of Ophthalmology. Oxford, U.K.: Oxford Univ. Press, 1999, vol. 2.

[5] C. Sinthanayothin, J. F. Boyce, H. L. Cook, and T. H.Williamson, "Automated localization of the optic disk, fovea, and retinal blood vessels from digital colour fundus images," Br. J. Ophthalmol., vol. 83, no. 8,pp. 902-910, 1999.

[6] H. Li and O. Chutatape, "Automatic location of optic disc in retinal images," in IEEE Int. Conf. Image Process., Oct. 7-10, 2001, vol. 2,pp. 837-840.

[7] T.Walter and J.-C. Klein, "Segmentation of color fundus images of the human retina: Detection of the optic disc and the vascular tree using morphological techniques," in Proc. 2nd Int. Symp. Med. Data Anal.,2001, pp. 282287.

[8] M. D. Abramoff, W. L. M. Alward, E. C. Greenlee, L. Shuba, C. Y. Kim, J. H. Fingert, and Y. H. Kwon,"Automated segmentation of the optic nerve head from stereo color photographs using biologically plausible feature detectors." Investigative Ophthalmology and Visual Sciences [in press], 2007.

[9] M. B. Merickel, X. Wu, M. Sonka, and M. D. Abramoff, "Optimal segmentation of the optic nerve head from stereo retinal images," Proceedings of SPIE Medical Imaging 6143, 2006.

[10] M. Sonka, V. Hlavac, and R. Boyle, Image Processing, Analysis, and Machine Vision, Brooks/Cole Publishing Company, second ed., 1999.

[11] J. Lowell, A. Hunter, D. Steel, A. Basu, R. Ryder, and E. Fletcher, "Optic nerve head segmentation," IEEE
Transactions on Medical Imaging 23(2), pp. 256-264, 2004.

[12] Morales, S., Naranjo, V., Angulo, J., and Alcañiz, M., 2013. Automatic Detection of Optic Disc Based on PCA and Mathematical Morphology. IEEE Trans. Med. Imag. 32, 786-796.

[13] Dashtbozorg Behdad, Ana Maria Mendonça, Aurélio Campilho, 2015. Optic disc segmentation using the sliding band filter.Computers in Biology and Medicine, 56, 1-12.

[14] Walter T, Massin P, Erginay A, Ordonez R, Jeulin C, Klein JC. Automatic detection of microaneurysms in color fundus images. Med Image Anal. 2007;11:555-66

[15] Hooshiar Zolfagharnasab, Ahmad Reza Naghsh-Nilchi Cauchy based matched filter for retinal vessels detection J Med Signals Sens, 4 (1) (2014), p. 1.

[16] M. R. N. Tagore, G. B. Kande, E. V. K. Rao, and B. P. Rao, Segmentation of retinal vasculature using phase congruency and hierarchical clustering. In: Proceedings of the $2^{\text {nd }}$ International Conference on Advances in Computing, Communications and Informatics (ICACCI 2013). 361-366, 2013.

[17] Aliaa Abdel-Haleim Abdel-Razik Youssif, Atef Zaki Ghalwash, And Amr Ahmed Sabry Abdel-Rahman Ghoneim, "Optic Disc Detection From Normalized Digital Fundus Images By Means Of A Vessels' Direction Matched Filter", IEEE Transactions On Medical Imaging, Vol. 27, No. 1, January 2008

[18] Li Chunming, Chiu-Yen Kao, John C. Gore, and Zhaohua Ding, 2007. Implicit Active Contours Driven by Local Binary Fitting Energy. In: Proceedings of IEEE Conference on Computer Vision and Pattern Recognition, C-VPR 2007,1 - 7.

[19] K. Zhang, H. Song, and L. Zhang, "Active contours driven by local image fitting energy," Pattern Recognition, vol. 43, no. 4, pp. 1199-1206, 2010.

[20] T. Kauppi, V. Kalesnykiene, J.K. Kamarainen, L. Lensu, I. Sorri, H. Uusitalo, H. Kalviainen, J. Pietila, DIARETDB0: Evaluation Database and Methodology for Diabetic Retinopathy Algorithms, Technical Report, 2005.

[21] Fumero, F., Alayon, S., Sanchez, J. L., Sigut, J., and Gonzalez-Hernandez, M., 2011. RIM-ONE: An open retinal image database for optic nerve evaluation. In: 24th International Symposium on Computer-Based Medical Systems (CBMS 2011), 1-6.

[22] G. D. Joshi, J. Sivaswamy, K. Karan, and R. Krishnadas, "Optic disk and cup boundary detection using regional information," Proc. IEEE International Symposium on Biomedical Imaging (ISBI), pp. 948-951,2010.

[23] PS Mittapalli, GB Kande, "Segmentation of optic disk and optic cup from digital fundus images for the assessment of glaucoma" Biomedical Signal Processing and Control, Vol 24, pp 34-46,2016. 